\title{
OPEN BOOKS AND THE WEINSTEIN CONJECTURE
}

\author{
MAX DÖRNER, HANSJÖRG GEIGES, AND KAI ZEHMISCH
}

\begin{abstract}
We show the existence of a contractible periodic Reeb orbit for any contact structure supported by an open book whose binding can be realised as a hypersurface of restricted contact type in a subcritical Stein manifold. A key ingredient in the proof is a higher-dimensional version of Eliashberg's theorem about symplectic cobordisms from a contact manifold to a symplectic fibration.
\end{abstract}

\section{INTRODUCTION}

A contact manifold $(M, \xi)$ is said to satisfy the Weinstein conjecture 28 if for every contact form $\alpha$ defining $\xi=\operatorname{ker} \alpha$ the corresponding Reeb vector field $R_{\alpha}$ has a closed orbit, cf. 24]. In a seminal paper, Hofer [15] developed a method to approach this conjecture in dimension 3 via the study of holomorphic discs in the symplectisation of $(M, \xi)$. This allowed him to prove the conjecture for overtwisted contact 3-manifolds and contact structures on 3-manifolds with nonvanishing second homotopy group. In [10] we explored the ramifications of Hofer's method in dimension 3 (including applications to symplectic 4-manifolds). In [11] we extended this approach to higher dimensions. Using a capping construction that can be traced back to McDuff 23 we were able to work with holomorphic spheres rather than discs, which simplifies the holomorphic analysis.

In dimension 3 the Weinstein conjecture has now been answered in the affirmative by Taubes [26, cf. [16, with the help of Seiberg-Witten theory. Prior to the work of Taubes, arguably the most important advance on the 3-dimensional Weinstein conjecture had been due to Abbas-Cieliebak-Hofer [1, who established the conjecture for all contact structures supported (in the sense of Giroux [13]) by a planar open book.

This paper continues the programme begun in [11 and is close in spirit to the work of Abbas et al., in the sense that we establish the Weinstein conjecture for contact structures (in dimension $\geq 5$ ) supported by suitable open books. Specifically, the property we require is that the binding of the open book be realisable as a hypersurface of restricted contact type in a subcritical Stein manifold, see Theorem 1. (Recall that a Stein manifold of real dimension $2 n$ has the homotopy type of an $n$-dimensional complex. It is called subcritical if its handle decomposition only contains handles up to dimension $n-1$.) According to Giroux, any compact contact manifold $\left(M^{2 n+1}, \xi\right)$ admits a supporting open book whose pages are Stein manifolds. Thus, the assumptions of our existence theorem for periodic Reeb orbits will in particular be satisfied if the pages are subcritical.

2010 Mathematics Subject Classification. 53D35; 37C27, 37J45, 57R17. 


\section{THE MAIN THEOREM}

Let $M$ be a closed, oriented manifold of dimension $2 n+1 \geq 5$ admitting a (cooriented) contact structure $\xi=\operatorname{ker} \alpha$, i.e. $\alpha \wedge(d \alpha)^{n}>0$. The Reeb vector field $R$ of the contact form $\alpha$ is given by $R \downarrow d \alpha \equiv 0$ and $\alpha(R) \equiv 1$.

Recall that an open book decomposition of $M$ is a pair $(B, \theta)$ consisting of an oriented codimension 2 submanifold $B \subset M$ with trivial normal bundle and a locally trivial fibration $\theta: M \backslash B \rightarrow S^{1}$ given in a neighbourhood $B \times D^{2} \subset M$ of $B$ by the angular coordinate in the $D^{2}$-factor. The manifold $B$ is called the binding; the closures $\Sigma$ of the fibres of $\theta$ are referred to as the pages of the open book. The pages are oriented consistently with the orientation of their boundary $B$.

The contact structure $\xi$ is said to be supported [13] by the open book decomposition $(B, \theta)$ if a contact form $\alpha$ can be chosen such that

(ob-i) the 2-form $d \alpha$ induces a positive symplectic form on each fibre of $\theta$, and

(ob-ii) the 1-form $\alpha$ induces a positive contact form on $B$.

Theorem 1. Let $(M, \xi)$ be a contact manifold of dimension $\geq 5$ with a supporting open book $(B, \theta)$ whose binding $B$ (with the induced contact structure) embeds as a hypersurface of restricted contact type into a subcritical Stein manifold. Then any contact form defining $\xi$ has a contractible periodic Reeb orbit.

Note that the theorem claims the existence of a closed Reeb orbit for any contact form defining $\xi$, not only those adapted to the open book in the sense of (ob-i) and (ob-ii).

\section{A NEIGHBOURHOOD THEOREM}

Our aim in this section is to prove a neighbourhood theorem for the binding $B$ of an open book. So we consider contact structures on $B \times D^{2}$ that satisfy the compatibility conditions (ob-i) and (ob-ii) with the open book structure on this neighbourhood of the binding.

For the purposes of this section we call

$$
\Sigma_{\theta}:=\left\{\left(b ; r \mathrm{e}^{\mathrm{i} \theta}\right) \in B \times D^{2}: b \in B, 0 \leq r \leq 1\right\}
$$

the 'page', and we identify $B$ with $B \times\{0\} \subset B \times D^{2}$.

Proposition 2. Let $\alpha$ be a contact form on $B \times D^{2}$ with the following properties:

(i) The 1-form $\alpha_{B}:=\left.\alpha\right|_{T B}$ is a contact form on $B$.

(ii) For each $\theta \in[0,2 \pi)$, the 2 -form $\left.d \alpha\right|_{T \Sigma_{\theta}}$ is a symplectic form on $\Sigma_{\theta} \backslash B$.

(iii) With the orientations of $B$ and $\Sigma_{\theta}$ induced by $\alpha_{B}$ and $\left.d \alpha\right|_{T \Sigma_{\theta}}$, respectively, $B$ is oriented as the boundary of $\Sigma_{\theta}$.

Then for $\varepsilon>0$ sufficiently small there is a page-preserving embedding

$$
B \times D_{\varepsilon}^{2} \longrightarrow B \times D^{2}
$$

which is the identity on $B \times\{0\}$ and pulls back $\alpha$ - after an isotopic modification of $\alpha$ near $B \times\{0\}$ via forms satisfying (i) (up to constant scale) to (iii) - to a 1-form

$$
h_{1}(r) \alpha_{B}+h_{2}(r) d \theta
$$

satisfying

- $h_{1}(0)>0, h_{2}(r)=r^{2}$ near $r=0$,

- $h_{1}^{n-1}\left(h_{1} h_{2}^{\prime}-h_{1}^{\prime} h_{2}\right)>0$ (the contact condition), 
- $h_{1}^{\prime}<0$ for $r>0$ (the symplectic condition on the pages).

Proof. The idea of the proof (due to Giroux) is to identify a neighbourhood of the binding with $B \times D_{\varepsilon}^{2}$ in such a way that the radial direction corresponds to the (negative) characteristic direction in the pages.

Choose volume forms $\Omega_{\theta}$ on the pages $\Sigma_{\theta}$ compatible with the orientation of the pages, e.g.

$$
\Omega_{\theta}=-(\cos \theta d x+\sin \theta d y) \wedge \alpha_{B} \wedge\left(d \alpha_{B}\right)^{n-1} .
$$

Write $\beta_{\theta}:=\left.\alpha\right|_{T \Sigma_{\theta}}$ for the restriction of the contact form $\alpha$ to the page $\Sigma_{\theta}$. The characteristic vector field $X_{\theta}$ is the vector field tangent to $\Sigma_{\theta}$ defined by

$$
\left.X_{\theta}\right\lrcorner \Omega_{\theta}=-\beta_{\theta} \wedge\left(d \beta_{\theta}\right)^{n-1},
$$

see [12] and 9, Section 2.5.4]. By conditions (i) and (iii), this vector field $X_{\theta}$ is transverse to $B \subset \Sigma_{\theta}$ and points into the page.

Along the binding we have a whole $S^{1}$-family of vector fields, so it is not immediately clear that the flow of the $X_{\theta}$ defines a smooth embedding. To circumvent this problem, we replace the time $r$ flow of the $X_{\theta}$ by the time 1 flow of a smooth $D^{2}$-family of 'honest' vector fields on $B \times D^{2}$. This was suggested to us by Fan Ding.

Write the points in the parameter space $D^{2}$ in polar coordinates as $R \mathrm{e}^{\mathrm{i} \varphi}$. Define a smooth $D^{2}$-family of smooth vector fields on $B \times D^{2}$ by

$$
\left.\tilde{X}_{R \mathrm{e}^{\mathrm{i} \varphi}}\right\lrcorner\left(d x \wedge d y \wedge \alpha_{B} \wedge\left(d \alpha_{B}\right)^{n-1}\right)=(R \sin \varphi d x-R \cos \varphi d y) \wedge \alpha \wedge(d \alpha)^{n-1} .
$$

We claim that

$$
\tilde{X}_{\left.R \mathrm{e}^{\mathrm{i} \theta}\right|_{\Sigma_{\theta}}}=R X_{\theta}
$$

Indeed, by taking the wedge product of the defining equation for $X_{\theta}$ with $\sin \theta d x-$ $\cos \theta d y$ and observing that

$$
T \Sigma_{\theta} \subset \operatorname{ker}(\sin \theta d x-\cos \theta d y)
$$

we have

$$
\left.X_{\theta}\right\lrcorner\left(d x \wedge d y \wedge \alpha_{B} \wedge\left(d \alpha_{B}\right)^{n-1}\right)=(\sin \theta d x-\cos \theta d y) \wedge \alpha \wedge(d \alpha)^{n-1},
$$

from which the claim follows.

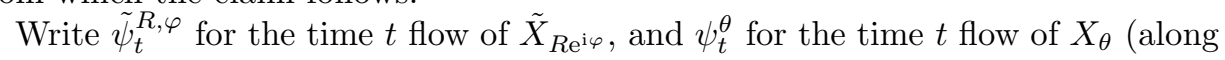
$\left.\Sigma_{\theta}\right)$. For $\varepsilon>0$ sufficiently small, this defines an embedding

$$
\Psi: \quad \begin{aligned}
B \times D_{\varepsilon}^{2} & \longrightarrow \quad B \times D^{2} \\
\left(b, r \mathrm{e}^{\mathrm{i} \theta}\right) & \longmapsto \tilde{\psi}_{1}^{r, \theta}(b, 0),
\end{aligned}
$$

where $D_{\varepsilon}^{2}$ denotes the 2 -disc of radius $\varepsilon$.

We maintain that

$$
\operatorname{ker}\left(\Psi^{*} \alpha\right)=\operatorname{ker}\left(\alpha_{B}+h d \theta\right)
$$

for some smooth function $h$ on $B \times D_{\varepsilon}^{2}$ that makes the 1-form $h d \theta$ well defined (in particular, $h=0$ along $B$ ) and smooth. Indeed, a straightforward computation, cf. [9. Lemma 2.5.20], gives

$$
X_{\theta} \in \operatorname{ker} \beta_{\theta} \quad \text { and } \quad L_{X_{\theta}} \beta_{\theta}=\mu_{\theta} \beta_{\theta}
$$

for some smooth function $\mu_{\theta}: \Sigma_{\theta} \rightarrow \mathbb{R}$. Since

$$
\tilde{\psi}_{1}^{r, \theta}(b, 0)=\psi_{r}^{\theta}(b, 0),
$$


we have $T \Psi\left(\left.\partial_{r}\right|_{\Sigma_{\theta}}\right)=X_{\theta}$. So the first condition on $X_{\theta}$ means that $\Psi^{*} \alpha$ contains no $d r$-component; the second condition means that $\operatorname{ker}\left(\left.\Psi^{*} \alpha\right|_{T \Sigma_{\theta}}\right)$ is invariant under the flow of $\partial_{r}$. This proves the claim.

The standard Gray stability argument applied to the linear interpolation of the contact forms $\alpha_{B}+h d \theta$ and $\alpha_{B}+r^{2} d \theta$ gives a flow fixed at $B$ and tangent to the $\partial_{r}$-direction which isotopes the contact structure $\operatorname{ker}\left(\alpha_{B}+h d \theta\right)$ to $\operatorname{ker}\left(\alpha_{B}+r^{2} d \theta\right)$.

In sum, we have an embedding $B \times D_{\varepsilon}^{2} \rightarrow B \times D^{2}$ (possibly for some smaller $\varepsilon>0)$ that pulls back $\alpha$ to a 1 -form $f\left(\alpha_{B}+r^{2} d \theta\right)$ with a positive function $f$ satisfying $f \equiv 1$ along $B \times\{0\}$. Observe that conditions (ii) and (iii) are equivalent to $\partial f / \partial r<0$. For ease of notation, we continue to write $\alpha$ for this pulled-back form.

Now let $\lambda:[0, \varepsilon] \rightarrow[0,1]$ be a smooth function that is identically 1 near $r=0$ and identically 0 near $r=\varepsilon$. Replace $\alpha$ by

$$
\left(\lambda(r)\left(c-r^{2}\right)+(1-\lambda(r)) f\right)\left(\alpha_{B}+r^{2} d \theta\right)
$$

where $c$ is a positive constant. This new 1 -form coincides with $\alpha$ away from $B \times\{0\}$, and is of the desired form $h_{1}(r) \alpha_{B}+h_{2}(r) d \theta$ near $B \times\{0\}$. For $c$ sufficiently large, the coefficient function $\lambda(r)\left(c-r^{2}\right)+(1-\lambda(r)) f$ has negative $r$-derivative, so condition (ii) will be satisfied. The linear interpolation between $\alpha$ and this modified form is via forms satisfying (i) to (iii).

Remark 3. For the capping construction below it is convenient to make a specific choice of the functions $h_{1}, h_{2}$ subject to the following conditions:

(i) $h_{1}(r)=c \mathrm{e}^{-r^{2}}$ near $r=0$ for some constant $c$,

(ii) $h_{1}(r)=c \mathrm{e}^{-r}$ and $h_{2}(r)$ constant for $r$ near some $r_{0}>0$.

Figure 1 illustrates that for $c$ sufficiently large it is possible to find such a pair $\left(h_{1}, h_{2}\right)$ that can be interpolated to the given pair (drawn as a dashed line) via pairs satisfying the conditions stipulated in the preceding lemma.

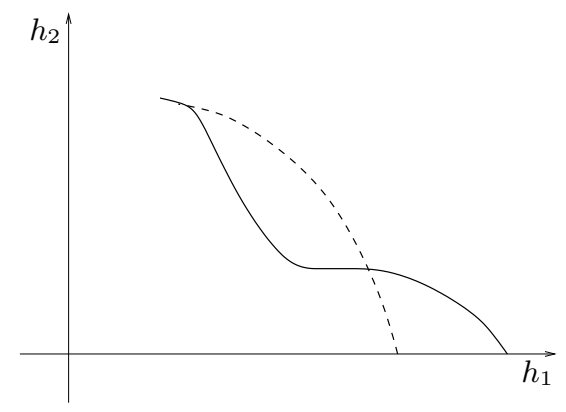

Figure 1. How to choose a specific $\left(h_{1}, h_{2}\right)$.

Remark 4. An open book can alternatively be described by a compact $2 n$-dimensional manifold $\Sigma$ with non-empty boundary $\partial \Sigma=: B$, and a monodromy diffeomorphism $\phi$ of $\Sigma$, equal to the identity near $\partial \Sigma$. The closed manifold $M$ obtained from the mapping cylinder of $(\Sigma, \phi)$ (which has boundary $\left.\partial \Sigma \times S^{1}\right)$ by attaching $\partial \Sigma \times D^{2}$ then has an open book decomposition $(B, \theta)$. Conversely, from $(B, \theta)$ one can recover $(\Sigma, \phi)$, cf. [9, Section 4.4.2]. 
A construction of Giroux [13], cf. [9, Section 7.3], produces a contact structure adapted to an open book, provided the page is exact symplectic with contact type boundary, and the monodromy is symplectic. Here the contact structure has, by construction, the normal form described in Proposition 2. So a consequence of the proposition is that any contact structure supported by an open book can be obtained via Giroux's construction.

\section{From OPEN BOOKS TO SYMPLECTIC FIBRATIONS}

The following theorem and its corollary are higher-dimensional analogues of 8 , Theorem 1.1], which was the crucial part in Eliashberg's construction of symplectic caps for weak symplectic fillings. Our proof is close in spirit to that of Eliashberg.

In the theorem and throughout this section, $(M, \xi=\operatorname{ker} \alpha)$ will be a closed contact manifold and $(B, \theta)$ an open book decomposition of $M$ to which the contact form $\alpha$ is adapted. We write $\Sigma$ for the page of the open book decomposition, and $\alpha_{B}$ for the restriction of $\alpha$ to $T B$.

Theorem 5. Suppose there is a (not necessarily compact) symplectic manifold $\left(C, \omega_{C}\right)$ with strong concave boundary that is strictly contactomorphic to $\left(B, \alpha_{B}\right)$.

Then there is a (not necessarily compact) symplectic manifold $(W, \omega)$ with boundary $\partial W=-M \sqcup N$, where $(M, \alpha)$ is a strong concave boundary component, and $N$ is a fibre bundle over $S^{1}$ with fibre $F=\Sigma \cup C$ such that $\omega$ restricts to a symplectic form on each fibre. Moreover, the holonomy of the fibre bundle $N \rightarrow S^{1}$ (given by the kernel of $\left.\left.\omega\right|_{T N}\right)$ is the identity on the subset $C \subset F$ of the fibre.

The condition on $C$ means that there is a Liouville vector field $Y$ defined near the boundary $\partial C$ and pointing inwards, such that $\left(-\partial C,\left.i_{Y} \omega_{C}\right|_{T(\partial C)}\right)$ is diffeomorphic to $\left(B, \alpha_{B}\right)$.

Corollary 6. Given any closed contact manifold $(M, \xi)$, there is a compact symplectic manifold $(W, \omega)$ with boundary $\partial W=-M \sqcup N$ such that $(M, \xi)$ is a strong concave boundary component and $\left(N,\left.\omega\right|_{T N}\right)$ is a symplectic fibre bundle over $S^{1}$.

Proof. By [13] we may assume that $(M, \xi)$ is supported by an open book whose page $\Sigma$ is a compact Stein manifold, i.e. a compact sublevel set of an exhausting, strictly plurisubharmonic function on a Stein domain. According to [22, Theorem 3.1], this Stein manifold $\Sigma$ embeds into a smooth projective variety. This means, in particular, that we find a compact symplectic cap $\left(C, \omega_{C}\right)$ for $\Sigma$ as required by Theorem 5

Proof of Theorem 5. Topologically, the definition of $W$ is very simple. Let $B \times D^{2} \subset$ $M$ be a neighbourhood of the binding as in Section 3. Then define

$$
W:=[-2,0] \times M \cup_{B \times D^{2}}\left(C \times D^{2}\right)
$$

(and smooth corners), where we think of $B \times D^{2}$ as, on the one hand, a subset of $M \times\{0\}$ and, on the other, as $\partial C \times D^{2}$. (We take a cylinder of height 2 over $M$ simply to have enough room for the construction that follows.)

Symplectically, we want to think of $[-2,0] \times M$ as a part of the symplectisation of $M$, i.e. we equip it with the symplectic form $d\left(\mathrm{e}^{s} \alpha\right)$. On $C \times D^{2}$ we take the symplectic form $\omega_{C}+f^{\prime}(\rho) d \rho \wedge d \theta$, where $(\rho, \theta)$ denote polar coordinates on $D^{2}$, and $f: \mathbb{R}_{0}^{+} \rightarrow \mathbb{R}_{0}^{+}$is a smooth function with the following properties:

(f-i) $f(\rho)=\rho^{2}$ near $\rho=0$ (so that the form above is smooth), 
(f-ii) $f^{\prime}(\rho)>0$ for $\rho>0$ (so that the form is symplectic),

(f-iii) $f(\rho)=\rho$ near $\rho=1$ (this will be relevant later, see condition (J1) in Section [5].

For the gluing, we work in the neighbourhood

$$
U:=[-1,0] \times B \times D^{2} \subset[-2,0] \times M,
$$

with polar coordinates $(r, \theta)$ on the $D^{2}$-factor. Purely for notational convenience, we shall assume that the contact form $\alpha$ is given on an open neighbourhood of $B \times D^{2}$ in $M$ by $h_{1}(r) \alpha_{B}+h_{2}(r) d \theta$ with

(h-i) $h_{1}(r)=\mathrm{e}^{-r^{2}}$ and $h_{2}(r)=r^{2}$ near $r=0$,

(h-ii) $h_{1}(r)=\mathrm{e}^{-r}$ and $h_{2}(r) \equiv 1$ near $r=1$.

By Remark 3, this can always be achieved (up to some inessential constants).

Both the gluing along parts of the boundary and the smoothing of corners are awkward processes in the presence of a symplectic (or any other geometric) structure. To avoid this, the idea is to construct a model which contains a symplectic copy both of $C \times D^{2}$ and $U$, and such that the identification of $[-2,0] \times M$ and this model along $U$ (or in fact a small open neighbourhood of it) realises the desired topological construction.

By the assumptions of the theorem, we obtain a symplectic manifold by gluing $(-\infty, 0] \times B$ and $C$ along $B \equiv\{0\} \times B \equiv-\partial C$. The model in question is the product of this manifold with a $D^{2}$-factor:

$$
\left(W_{0}, \omega_{0}\right):=\left(\left((-\infty, 0] \times B, d\left(\mathrm{e}^{t} \alpha_{B}\right)\right) \cup_{B}\left(C, \omega_{C}\right)\right) \times\left(D^{2}, f^{\prime}(\rho) d \rho \wedge d \theta\right),
$$

see Figure 2. In that figure, the left part of the horizontal axis represents $(-\infty, 0] \times$ $B$; the right part, $C$. The vertical axis gives the $\rho$-coordinate of the $D^{2}$-factor, so a 'realistic' picture is given by rotating the figure around the horizontal axis.

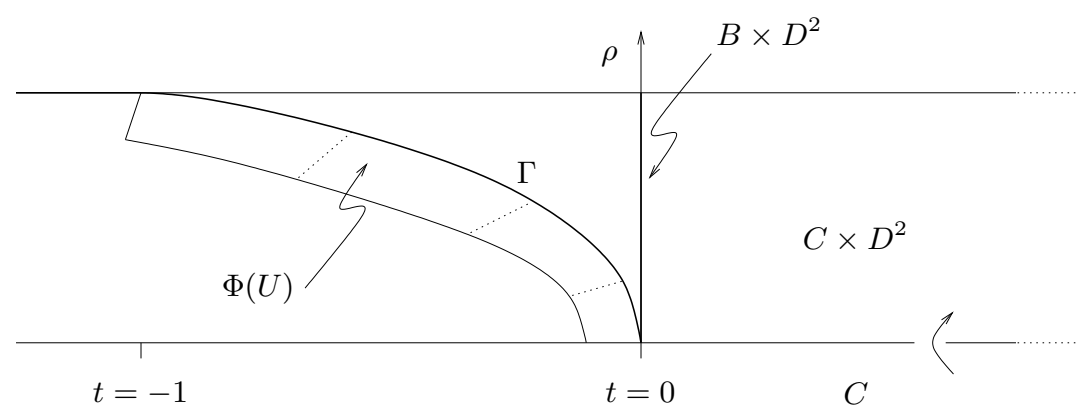

Figure 2. The model $\left(W_{0}, \omega_{0}\right)$ for the symplectic gluing.

We claim that we can find a symplectomorphic copy $\Phi(U)$ of $U$ in this model as indicated in the figure. The dotted lines represent flow lines of the Liouville vector field $T \Phi\left(\partial_{s}\right)$. The hypersurface $\Gamma$ in the model is then a strictly contactomorphic copy of $\left(B \times D^{2}, \alpha\right)$.

The trick, as it were, is to think of a neighbourhood of $B \times D^{2}$ in $[-2,0] \times M$ not as a neighbourhood to the left of the horizontal axis in the model (in which case the gluing would produce a corner at $\{0\} \times B \times\{\rho=1\})$, but as a neighbourhood below the 'curve' $\Gamma$ (which connects smoothly with the curve $\{\rho=1\}$ at $t=-1$ ). We can then glue the part of the model to the right of $\Gamma$ in a smooth and symplectic fashion to $[-2,0] \times M$. 
Write

$$
\lambda:=\mathrm{e}^{t} \alpha_{B}+f(\rho) d \theta
$$

for the primitive of $\omega_{0}$ on $(-\infty, 0] \times B \times D^{2} \subset W_{0}$. The corresponding Liouville vector field is

$$
Y=\partial_{t}+\frac{f(\rho)}{f^{\prime}(\rho)} \partial_{\rho}
$$

Now define

$$
\Phi: U=[-1,0] \times B \times D^{2} \longrightarrow(-\infty, 0] \times B \times D^{2} \subset W_{0}
$$

by

$$
(s, b, r, \theta) \longmapsto\left(s+\log \left(h_{1}(r)\right), b, f^{-1}\left(\mathrm{e}^{s} h_{2}(r)\right), \theta\right) .
$$

Lemma 7. The map $\Phi$ is a symplectic embedding with $\Phi^{*} \lambda=\mathrm{e}^{s} \alpha$ (and hence $\left.T \Phi\left(\partial_{s}\right)=Y\right)$.

Proof. Near $r=0$ we have $f^{-1}\left(\mathrm{e}^{s} h_{2}(r)\right)=\mathrm{e}^{s / 2} r$ by (f-i) and (h-i), so $\Phi$ is smooth.

In order to see that $\Phi$ is injective, assume that we have

$$
\Phi\left(s_{1}, b, r_{1}, \theta\right)=\Phi\left(s_{2}, b, r_{2}, \theta\right) .
$$

By looking at the first and third component of the image, we see that

$$
\mathrm{e}^{s_{1}} h_{1}\left(r_{1}\right)=\mathrm{e}^{s_{2}} h_{1}\left(r_{2}\right) \text { and } \mathrm{e}^{s_{1}} h_{2}\left(r_{1}\right)=\mathrm{e}^{s_{2}} h_{2}\left(r_{2}\right),
$$

whence

$$
\frac{h_{2}}{h_{1}}\left(r_{1}\right)=\frac{h_{2}}{h_{1}}\left(r_{2}\right) .
$$

By the contact condition, we have $\left(h_{2} / h_{1}\right)^{\prime}<0$. So this last equation forces $r_{1}=r_{2}$ and hence $s_{1}=s_{2}$.

The fact that $\Phi^{*} \lambda=\mathrm{e}^{s} \alpha$ follows from a quick calculation. In particular, this makes $\Phi$ symplectic, and hence an immersion.

The submanifold $\Gamma$ in Figure 2 is defined to be

$$
\Gamma:=\Phi\left(\{0\} \times B \times D^{2}\right) .
$$

By construction, this is a hypersurface of contact type (transverse to the Liouville vector field $Y$ ), and the induced contact form $\left.\lambda\right|_{T \Gamma}$ pulls back to $\alpha$ under the embedding $\Phi$.

Lemma 8. The hypersurface $\Gamma \subset(-\infty, 0] \times B \times D^{2}$ can be described as a graph

$$
\Gamma=\left\{(t, b, \rho, \theta) \in(-\infty, 0] \times B \times D^{2}: t \in[-1,0], \rho=g(t)\right\},
$$

where

$$
g(t):=f^{-1}\left(h_{2}\left(h_{1}^{-1}\left(\mathrm{e}^{t}\right)\right)\right) .
$$

Proof. The hypersurface $\Gamma$ is given by the $(t, b, \rho, \theta)$ with

$$
t=\log h_{1}(r) \text { and } \rho=f^{-1}\left(h_{2}(r)\right) .
$$

This clearly translates into the form in the lemma. 
Note that $g^{\prime}(t) \leq 0$. Near $t=0$ we have $g(t)=\sqrt{-t}$ by (f-i) and (h-i). This shows that $\Gamma$ looks like a 'paraboloid' near $t=0$. In particular, this verifies again the smoothness of $\Gamma$.

Near $t=-1$ we have $g \equiv 1$ by (h-ii) and (f-iii). This means that $\Gamma$ coincides with $(-\infty, 0] \times B \times \partial D^{2}$ near $t=-1$. Therefore, the part of $W_{0}$ to the right of $\Gamma$, which is diffeomorphic to $C \times D^{2}$, can be glued to $[-2,0] \times M$ along $\{0\} \times B \times D^{2}$, resulting in a symplectic manifold $(W, \omega)$ with (smooth) boundary the disjoint union of $\{-2\} \times M$ and

$$
N:=\left(M \backslash\left(B \times \operatorname{Int}\left(D^{2}\right)\right)\right) \cup_{B \times S^{1}}\left(\left(([-1,0] \times B) \cup_{B} C\right) \times S^{1}\right) .
$$

This manifold $N$ fibres in an obvious way over $S^{1}$, with fibre given by

$$
(\Sigma \backslash\{r<1\}) \cup([-1,0] \times B) \cup C,
$$

which topologically is just $\Sigma \cup C$. The restriction of $\omega$ to this fibre is given by $d \alpha$ on the first part, by $d\left(\mathrm{e}^{t} \alpha_{B}\right)$ on the second, and by $\omega_{C}$ on the third. So the fibre is indeed symplectic. Finally, the symplectic monodromy of the $S^{1}$-fibration

$$
\left(C \times S^{1}, \omega_{C}\right) \longrightarrow S^{1}
$$

is obviously the identity. This completes the proof of Theorem 5 ,

Remark 9. After this result was presented by one of us (M. D.) in our local Arbeitsgemeinschaft, an alternative proof was found by M. Klukas [17.

The open book fibration $\theta: M \backslash B \rightarrow S^{1}$ and the projection onto the angular coordinate $\theta$ in the $D^{2}$-factor of the model $W_{0}$ (outside $0 \in D^{2}$ ) induce a fibration over $S^{1}$ of a whole collar neighbourhood of $N$ in $W$. We continue to write $\theta$ for this fibration and $d \theta$ for the induced 1-form on this collar.

Recall that a stable Hamiltonian structure on a manifold $N$ of dimension $2 n+1$ is a pair $(\Omega, \gamma)$ consisting of a 2 -form $\Omega$ and a 1-form $\gamma$ such that $\gamma \wedge \Omega^{n} \neq 0$ and ker $\Omega \subset \operatorname{ker} d \gamma$, see [6]. The Reeb vector field $R$ of such a stable Hamiltonian structure is defined by $R \in \operatorname{ker} \Omega$ and $\gamma(R)=1$. The symplectisation of $(\Omega, \gamma)$ is the symplectic manifold $((-\varepsilon, \varepsilon) \times N, \Omega+d(\rho \gamma))$ with $\varepsilon>0$ sufficiently small.

With $N$ the manifold we just constructed, and $\omega_{N}:=\left.\omega\right|_{T N}$, the pair $\left(\omega_{N}, d \theta\right)$ is an example of such a stable Hamiltonian structure, as is clear from the symplectic fibration property of $N$. The following lemma says that the symplectic form $\omega$ looks like the symplectisation of $\left(\omega_{N}, d \theta\right)$ in a collar neighbourhood of $N$ in $W$.

Lemma 10. There is a collar neighbourhood $(-\varepsilon, 0] \times N$ of $N$ in $W$ on which the symplectic form $\omega$ can be written as

$$
\omega=\omega_{N}+d \rho \wedge d \theta
$$

where, by slight abuse of notation, $\rho$ denotes the collar parameter.

Proof. By condition (f-iii), the symplectic form looks as claimed near the part of $N$ made up of $[-1,0] \times B \times S^{1}$ and $C \times S^{1}$, with $\rho$ the radial parameter in the $D^{2}$-factor. Up to an additive constant, we may take this $\rho$ as the collar parameter. In that region $\omega_{N}$ equals $d\left(e^{t} \alpha_{B}\right)$ and $\omega_{C}$, respectively.

Define a non-vanishing vector field $X$ on a neighbourhood of $N \subset W$ by

$$
X\lrcorner \omega=d \theta .
$$

Then $L_{X} \omega=0$, so the flow of $X$ leaves $\omega$ invariant. Near the part of $N$ considered previously we have $X=\partial_{\rho}$. 
We claim that $X$ is transverse to $N$ everywhere. By what we have just observed, this is only an issue near the part $M \backslash\left(B \times D^{2}\right)$ of $N$, where $\omega_{N}=d \alpha$. By condition (ob-i), together with the orientation conditions, the Reeb vector field $R_{\alpha}$ of $\alpha$ (which coincides up to scale with the Reeb vector field $R$ of the stable Hamiltonian structure) is positively transverse to the fibres of the open book, hence

$$
\omega(X, R)=d \theta(R)>0 .
$$

On the other hand, if $X$ were tangent to $N \subset \partial W$ at some point of $M \backslash\left(B \times D^{2}\right) \subset$ $N$, then at that point we would have

$$
\omega(X, R)=\omega_{N}(X, R)=d \alpha(X, R)=0,
$$

so that cannot happen.

Now define the collar parameter $\rho$ by the flow of $X$. Then, by the defining equation for $X$ and the invariance of $\omega$, the description of $\omega$ is as claimed in the lemma.

\section{Proof of Theorem 1}

The proof of Theorem 1 is based on the study of holomorphic curves in a symplectic cobordism having $(M, \xi)$ as its concave end. For the construction of this cobordism we rely on Theorem 5 , where we made a specific choice of contact form $\alpha$. Nonetheless, our main theorem holds for any contact form defining $\xi$, since up to a constant factor any such form can be realised on the concave end of a suitable symplectic cobordism by adding a negative half-symplectisation to the cobordism we are about to construct and choosing a suitable graph-like hypersurface in that cylindrical end, cf. [10, Remark 3.10] or [11, p. 265].

At some point in the proof of Theorem 1 we appeal to a compactness theorem from symplectic field theory, which presupposes the contact form defining $\xi$ to be non-degenerate. Again, this is not a restriction on the allowable contact forms, since the degenerate case follows from the non-degenerate one by an Arzelà-Ascoli type argument as in [1], cf. [10, Remark 6.4] or [11, Section 6.4].

5.1. Construction of the cobordism. By assumption, the binding $(B, \xi \cap T B)$ embeds into a subcritical Stein manifold as a hypersurface of restricted contact type. According to a result of Cieliebak [4, cf. [5, Theorem 14.16], any such subcritical Stein manifold is symplectomorphic to a split one $\left(V \times \mathbb{C}, J_{V} \oplus i\right)$, so we may think of $B$ as a hypersurface in $V \times \mathbb{C}$. On that split Stein manifold we have a strictly plurisubharmonic function $\psi=\psi_{V}+|z|^{2} / 4$, with $\psi_{V}$ strictly plurisubharmonic on the Stein manifold $V$. Choose a regular level set $S:=\psi^{-1}(c)$ of $\psi$ such that $B$ is contained in the interior of the corresponding sublevel set. Write $A_{B S}$ for the resulting cobordism between $B$ and $S$.

The condition on $B$ being of restricted contact type tells us that there is a global Liouville vector field on the symplectic manifold $V \times \mathbb{C}$ transverse to $B$ and inducing a contact form for $\xi \cap T B$ there. (The Liouville vector field necessarily points into $A_{B S}$ along $B$, see the proof of [11, Corollary 4.2], but it need not be transverse to $S$.) By adding a half-symplectisation along $B$ and taking a graph in that halfsymplectisation - analogous to what we said at the beginning of this section we obtain a cobordism (which we continue to call $A_{B S}$ ) with an exact symplectic form $d \mu$ such that $\mu=\mathrm{e}^{t} \alpha_{B}$ near $B$, up to constant scale, with $t \in[0, \varepsilon)$, say. This 
$d \mu$ is the symplectic form coming from the Stein structure away from the collar we added to $B$, but $\mu$ need not induce a contact structure on $S$.

In [11] we constructed a (compact, but non-closed!) symplectic cap $\left(C_{\infty}, \omega_{\infty}\right)$ for a hypersurface $S$ of the type described above. We adapt this construction to the present setting. Here we shall even work with a non-compact cap. Since the behaviour of holomorphic spheres near the boundary resp. in the non-compact end is well controlled, this issue is inessential.

Thus, we form the symplectic manifold $\left(C_{\infty}, \omega_{\infty}\right)$ by compactifying the $\mathbb{C}$-factor in $\psi^{-1}([c, \infty))$ to a $\mathbb{C P}^{1}$ with a scaled Fubini-Study form. Note that $C_{\infty}$ contains the complex hypersurface $V_{\infty}:=V \times\{\infty\}$. We then glue this with the described cobordism to obtain the symplectic manifold

$$
\left(C, \omega_{C}\right):=\left(A_{B S}, d \mu\right) \cup_{S}\left(C_{\infty}, \omega_{\infty}\right)
$$

with $\left(B, \alpha_{B}\right)$ as the strong concave boundary. This places us in the setting of Theorem 5

Moreover, the primitive $\lambda$ of $\omega_{0}$ on $(-\infty, 0] \times B \times D^{2}$, introduced in the proof of Theorem [5] extends to a primitive on $A_{B S} \times D^{2}$, which will be relevant for the subsequent analysis of holomorphic curves (specifically, the proof of Lemma 13).

Now construct $(W, \omega)$ as in Theorem 5 , and complete this symplectic manifold at the negative end, i.e. form the manifold

$$
\widetilde{W}:=(-\infty,-2] \times M \cup_{M} W
$$

with symplectic form

$$
\tilde{\omega}:= \begin{cases}d\left(\mathrm{e}^{s} \alpha\right) & \text { on }(-\infty,-2] \times M, \\ \omega & \text { on } W .\end{cases}
$$

5.2. The almost complex structure on $\widetilde{W}$. On the symplectic manifold $(\widetilde{W}, \tilde{\omega})$ we choose an almost complex structure $J$ tamed by $\tilde{\omega}$. Outside the collar $[-\varepsilon / 2,0] \times$ $N$ and $C_{\infty} \times D^{2}$, the almost complex structure may be chosen generically. Inside these regions, we impose the following conditions. Recall that we write $R$ for the Reeb vector field of the stable Hamiltonian structure $\left(\omega_{N}, d \theta\right)$ on the collar $(-\varepsilon, 0] \times N$, i.e. $R\lrcorner \omega_{N}=0$ and $d \theta(R)=1$, see Lemma 10. Also recall that on $C \times S^{1} \subset N$ the $S^{1}$-fibration of $N$ is the obvious one, and the restriction of $\omega_{N}$ to the fibres $C$ equals $\omega_{C}$.

By $J_{\infty}$ we denote the complex structure on $C_{\infty}$ coming from the inclusion $C_{\infty} \subset$ $V \times \mathbb{C P}^{1}$.

(J1) Over $C_{\infty} \times D^{2}$ we take $J$ to be a split structure $J_{\infty} \oplus j$, where $j$ is given by $j\left(\partial_{\rho}\right)=\partial_{\theta}$ for $\rho \in[1-\varepsilon / 2,1]$. (Here we rely on condition (f-iii).)

(J2) Over $[-\varepsilon / 2,0] \times N$ we take $J$ to respect the splitting $\operatorname{ker} d \theta \oplus\left\langle\partial_{\rho}, R\right\rangle$ (so that in particular the fibres of each $\{\rho\} \times N$ are holomorphic); on $\left\langle\partial_{\rho}, R\right\rangle$ we require $J\left(\partial_{\rho}\right)=R$; on ker $d \theta$ we choose $J$ compatible with $\left.\omega_{N}\right|_{\operatorname{ker} d \theta}$.

(J3) On the cylindrical end $(-\infty, 0] \times M$, the almost complex structure $J$ is cylindrical and symmetric in the sense of $[3$, i.e. it preserves $\xi=\operatorname{ker} \alpha$ and satisfies $J \partial_{s}=R_{\alpha}$.

Note that on the overlap of the two regions specified in (J1) and (J2) we have $R=\partial_{\theta}$, so an almost complex structure satisfying (J1) automatically satisfies (J2). By Lemma 10, an almost complex structure satisfying (J2) is compatible with $\omega$ on the collar $[-\varepsilon / 2,0] \times N$. 
5.3. Holomorphic spheres in $(\widetilde{W}, J)$. We now recall from [1] the essential information about holomorphic spheres in $C_{\infty}$. As in that paper, we consider a closed neighbourhood $U_{\partial}$ of the positive end of $\widetilde{W}$, defined by

$$
U_{\partial}:=\left\{x \in V: \psi_{V}(x) \geq c\right\} \times \mathbb{C P}^{1} \times D^{2} \subset C_{\infty} \times D^{2} \subset \widetilde{W},
$$

where $c$ is the level used in the definition of $S=\psi^{-1}(c)$. Observe that $U_{\partial}$ is foliated by holomorphic spheres $\{p\} \times \mathbb{C P}^{1} \times\{w\}$.

Lemma 11. Let $u: \mathbb{C P}^{1} \rightarrow \widetilde{W}$ be a smooth non-constant $J$-holomorphic sphere.

(i) If $u\left(\mathbb{C P}^{1}\right) \cap\left(C_{\infty} \times D^{2}\right) \neq \emptyset$, then $u\left(\mathbb{C P}^{1}\right) \cap\left(V_{\infty} \times D^{2}\right) \neq \emptyset$.

(ii) If $u\left(\mathbb{C P}^{1}\right) \cap U_{\partial} \neq \emptyset$, then $u\left(\mathbb{C P}^{1}\right) \subset U_{\partial}$, and $u$ is of the form $z \mapsto$ $(p, v(z), w)$, where $v$ is some holomorphic covering of $\mathbb{C P}^{1}$ by itself.

(iii) If $u\left(\mathbb{C P}^{1}\right) \subset C_{\infty} \times D^{2}$, then $u$ is one of the spheres in (ii).

The proof is completely analogous to that of [11, Lemma 5.2].

5.4. The moduli space of holomorphic spheres. Fix a holomorphic sphere

$$
F:=\left\{p_{0}\right\} \times \mathbb{C P}^{1} \times\left\{w_{0}\right\} \subset U_{\partial},
$$

where the choice is made such that $F$ is not contained in the collar neighbourhood $[-\varepsilon / 2,0] \times N$. Write $\widetilde{\mathcal{M}}$ for the moduli space of smooth $J$-holomorphic spheres $u: \mathbb{C P}^{1} \rightarrow \widetilde{W}$ representing the class $[F] \in H_{2}(\widetilde{W})$ and not contained in $[-\varepsilon / 2,0] \times N$. This last condition we impose to avoid problems with transversality caused by the non-generic almost complex structure on the collar. The homological intersection number of $[F]$ with the class in $H_{2 n}\left(\widetilde{W}, U_{\partial} \cup C_{\infty} \times \partial D^{2}\right)$ represented by the complex hypersurface $V_{\infty} \times D^{2}$ equals 1 .

In conjunction with Lemma 11] we see that any holomorphic sphere that touches $U_{\partial}$ or is contained in $C_{\infty} \times D^{2}$ is of the form $z \mapsto(p, v(z), w)$ for some $v \in \operatorname{Aut}\left(\mathbb{C P}^{1}\right)$. This tells us that all holomorphic spheres corresponding to points in $\widetilde{\mathcal{M}}$ near its ends are standard.

As in [11, Proposition 6.1], we have the following statement.

Proposition 12. The moduli space $\widetilde{\mathcal{M}}$ is a smooth manifold of dimension $2 n+6$, and all elements of $\widetilde{\mathcal{M}}$ are simple holomorphic spheres.

The spheres in $\widetilde{\mathcal{M}}$ being simple implies that the quotient space

$$
\mathcal{M}:=\widetilde{\mathcal{M}} \times \operatorname{Aut}\left(\mathbb{C P}^{1}\right) \mathbb{C P}{ }^{1}
$$

is a smooth manifold of dimension $2 n+2$.

5.5. Spheres intersecting an arc. Let $\gamma$ be a proper embedding of the interval $[0,1)$ into $\widetilde{W}$ with $\gamma(0) \in F$ and $\gamma(t) \in(-\infty,-2] \times M$ for $t$ near 1 . In addition, we require that the image of $\gamma$ lie in the complement of the collar neighbourhood $[-\varepsilon / 2,0] \times N$. This ensures that the space

$$
\mathcal{M}_{\gamma}:=\mathrm{ev}^{-1}(\gamma)
$$

where ev is the evaluation map

$$
\text { ev: } \begin{array}{clc}
\mathcal{M} & \longrightarrow \widetilde{W} \\
{[u, z]} & \longmapsto u(z),
\end{array}
$$

is a smooth 1-dimensional manifold (with boundary). 
Any sphere in $\mathcal{M}$ represents the class $[F]$, which has homological intersection number zero with any fibre of $N$. Thus, by positivity of intersection [7, Proposition 7.1], any sphere in $\mathcal{M}$ is either disjoint from the collar $[-\varepsilon / 2,0] \times N$ or contained in a single fibre of some $\{\rho\} \times N$. Therefore, our choice of $\gamma$ ensures that no sphere in $\mathcal{M}_{\gamma}$ can touch the collar.

Near the preimage of $\gamma(0)$, the manifold $\mathcal{M}_{\gamma}$ looks like a half-open interval, but there are no other boundary points. In particular, $\mathcal{M}_{\gamma}$ is non-compact.

5.6. The conclusion of the argument. By the non-compactness of $\mathcal{M}_{\gamma}$ we find a sequence of points in $\mathcal{M}_{\gamma}$ without any convergent subsequence. The compactness theorem from symplectic field theory [3. Theorem 10.2], however, implies the existence of a subsequence convergent in the Gromov-Hofer sense to a holomorphic building of height $k_{-} \mid 1$. We want to show that $k_{-}>0$, which is equivalent to saying that the subsequence is not Gromov-convergent to a bubble tree in $\widetilde{W}$.

By construction, the symplectic form $\tilde{\omega}$ is exact on $\widetilde{W} \backslash\left(C_{\infty} \times D^{2}\right)$. Hence, any non-constant sphere in a purported bubble tree must intersect $C_{\infty} \times D^{2}$, and further, by Lemma 11 (i), each sphere intersects the complex hypersurface $V_{\infty} \times D^{2}$. By positivity of intersection [7] and the homological intersection of $F$ with $V_{\infty} \times D^{2}$ being 1, there can be no non-trivial bubble trees. So, indeed, a non-convergent sequence in $\mathcal{M}_{\gamma}$ has to break into a building with $k_{-}>0$.

The proof of Theorem 1 is completed with the following lemma.

Lemma 13. The described building contains a finite energy plane with a positive puncture, corresponding to a contractible Reeb orbit.

Proof. In any holomorphic building of height $k_{-} \mid 1$ with $k_{-}>0$ there must be at least two finite energy planes. By our intersection argument, at most one of these can intersect $V_{\infty} \times D^{2}$. Any other finite energy plane in the top level $W$ of the building would have to stay outside $C_{\infty} \times D^{2}$. This, however, is impossible, because there the symplectic form $\tilde{\omega}$ is exact (see the comment in Section [5.1 right before the construction of $\widetilde{W}$ ), so by Stokes's theorem the Hofer energy of a finite energy plane with a negative end would be negative, cf. [3. Lemma 5.16]. Likewise, there can be no finite energy plane with a negative puncture in any of the lower levels of the building, all of which are copies of the symplectisation of $M$ (and hence exact). We conclude that there must be a finite energy plane with a positive puncture in one of the lower levels $\left(\mathbb{R} \times M, d\left(\mathrm{e}^{s} \alpha\right)\right)$ of the building.

\section{ExAmples}

In this section we discuss some applications and mild extensions of our main theorem. The following list is meant to be illustrative rather than exhaustive.

(1) The contrapositive of our theorem allows us to draw topological conclusions. Given a contact manifold $(M, \xi=\operatorname{ker} \alpha)$ with $R_{\alpha}$ not having any contractible periodic Reeb orbit, one can deduce that $\xi$ is not supported by an open book with subcritical pages. Examples are provided by the unit cotangent bundle of any $n$ torus $\mathbb{R}^{n} / \Lambda$ obtained as a quotient of $\mathbb{R}^{n}$ with the euclidean metric, since the Reeb flow on the unit cotangent bundle of any Riemannian manifold coincides with the cogeodesic flow, cf. 9, Theorem 1.5.2].

(2) In our construction, $A_{B S}$ was a symplectic cobordism with an exact symplectic form. Under the weaker assumption that $A_{B S}$ is any semi-positive symplectic 
cobordism, one can still infer the existence of at least a nullhomologous Reeb link as follows.

As in the proof of Theorem 1, we need only exclude Gromov-convergent sequences. Any potential bubble tree arising as a Gromov limit of a sequence in $\mathcal{M}_{\gamma}$ has to stay outside the collar $[-\varepsilon / 2,0] \times N$ by an intersection argument. Indeed, at least one sphere in the bubble intersects $\gamma$ and hence is not contained entirely in the collar. If the bubble tree were to intersect the collar, there would be at least one sphere having positive intersection with a fibre of (a copy of) $N$. On the other hand, we can obviously find a different fibre (in a different copy of $N$ ) having empty intersection with the bubble tree.

Provided we can show that the cobordism $\widetilde{W} \backslash\left(C_{\infty} \times D^{2}\right)$ is semi-positive, we may then appeal to [11, Section 6.3] to rule out non-trivial bubble trees.

For $n=2$, semi-positivity of this 6-dimensional cobordism is automatic. For $n \geq 3$, we argue as follows. Recall that

$$
\widetilde{W} \backslash\left(C_{\infty} \times D^{2}\right)=(-\infty, 0] \times M \cup_{B \times D^{2}}\left(A_{B S} \times D^{2}\right),
$$

which is homotopy equivalent to $M \cup_{B \times D^{2}}\left(A_{B S} \times D^{2}\right)$. We have

$$
H_{2}\left(M \backslash B \times \operatorname{Int}\left(D^{2}\right), B \times S^{1}\right) \cong H^{2 n-1}\left(M \backslash B \times \operatorname{Int}\left(D^{2}\right)\right)=0 \text { for } n \geq 3,
$$

since $M \backslash B$ fibres over $S^{1}$ with fibres having the homotopy type of an $n$-dimensional complex. This implies that any class in $H_{2}\left(\widetilde{W} \backslash\left(C_{\infty} \times D^{2}\right)\right)$ is homologous to one in $A_{B S} \times D^{2} \simeq A_{B S}$. Moreover, $A_{B S}$ is a complex hypersurface in $A_{B S} \times D^{2}$, so the values of $[\tilde{\omega}]$ and $c_{1}(\widetilde{W}, J)$, respectively, on any homology class in $H_{2}\left(\widetilde{W} \backslash\left(C_{\infty} \times D^{2}\right)\right)$ can be computed inside $A_{B S}$, where semi-positivity holds.

(3) A Lagrangian embedding of the 2-sphere into $\mathbb{C}^{2}$ blown up in two points can be defined as follows, cf. [25. Example 2.14]. Start with the Lagrangian cylinder

$$
\left\{\left(r, \mathrm{e}^{\mathrm{i} \varphi}\right) \in \mathbb{C}^{2}: r \in[0,3], \varphi \in[0,2 \pi)\right\} .
$$

Now form a symplectic blow-up by cutting out the open unit ball in $\mathbb{C}^{2}$ centred at $(0,0)$ and collapsing the fibres of the Hopf fibration on the boundary sphere. We maintain that the cylinder descends to a Lagrangian disc in the blown-up manifold. A similar blow-up centred at $(3,0)$ then produces a Lagrangian 2 -sphere in $\mathbb{C}^{2} \# \overline{\mathbb{C P}}^{2} \# \overline{\mathbb{C P}}^{2}$.

The most convenient way to see that the cylinder descends to a smooth disc in the blown-up manifold is Lerman's description of a blow-up as a symplectic cut 21. That is, on $\mathbb{C}^{2} \times \mathbb{C}$ with coordinates $\left(z_{1}, z_{2}, w\right)$ and standard symplectic form we consider the $S^{1}$-action given by

$$
\mathrm{e}^{\mathrm{i} \theta}\left(z_{1}, z_{2}, w\right)=\left(\mathrm{e}^{\mathrm{i} \theta} z_{1}, \mathrm{e}^{\mathrm{i} \theta} z_{2}, \mathrm{e}^{-\mathrm{i} \theta} w\right) .
$$

The function $\psi: \mathbb{C}^{2} \times \mathbb{C} \rightarrow \mathbb{R}$ given by $\psi\left(z_{1}, z_{2}, w\right)=\left|z_{1}\right|^{2}+\left|z_{2}\right|^{2}-|w|^{2}$ is $S^{1}$ invariant, and so is the symplectic form. The blown-up manifold is the smooth symplectic quotient of the level set $\psi^{-1}(1)$ under the free $S^{1}$-action.

The isotropic cylinder

$$
\left\{\left(r, \mathrm{e}^{\mathrm{i} \varphi}, r\right) \in \mathbb{C}^{2} \times \mathbb{C}: r \in[0,3], \varphi \in[0,2 \pi)\right\} \subset \psi^{-1}(1)
$$

descends to a disc in the blown-up manifold which is Lagrangian outside its centre. Provided the disc is smooth at the centre, it will be Lagrangian by continuity. We claim that

$$
(r, \varphi) \longmapsto\left[r: \mathrm{e}^{\mathrm{i} \varphi}: r\right]
$$


defines a smooth parametrisation of the disc. Beware that the 'homogeneous' coordinates here refer to the quotient under the $S^{1}$-action only. Indeed, we have

$$
\left[r: \mathrm{e}^{\mathrm{i} \varphi}: r\right]=\left[r \mathrm{e}^{-\mathrm{i} \varphi}: 1: r \mathrm{e}^{\mathrm{i} \varphi}\right],
$$

so the parametrisation can be rewritten as $z \mapsto[\bar{z}: 1: z]$.

Now, by Weinstein's neighbourhood theorem for Lagrangian submanifolds [27, a small cotangent disc bundle of $S^{2}$ embeds symplectically into $\mathbb{C}^{2} \# \overline{\mathbb{C P}}^{2} \# \overline{\mathbb{C P}}^{2}$. This gives us a symplectic cobordism from $\mathbb{R P}^{3}$ to $S^{3}$ (with their standard contact structures), which is semi-positive for dimensional reasons.

Then Example (2) applies to show that the contact manifold obtained from an open book whose page is the cotangent disc bundle of $S^{2}$, and whose monodromy is a $k$-fold Dehn twist $\tau^{k}, k \in \mathbb{Z}$, carries a nullhomologous Reeb link. For $k \geq 0$ the resulting manifolds are the 5 -dimensional Brieskorn manifolds $\Sigma(k, 2,2,2)$ with their natural contact structure, see [20]; for $k<0$ we obtain a contact structure on a diffeomorphic copy of $\Sigma(|k|, 2,2,2)$. Since these manifolds are simply connected, see [14, we obviously get a contractible periodic Reeb orbit. The reader may wish to compare this with the results of van Koert [19.

It was shown by Seidel [25], Proposition 2.4] that the Dehn twists $\tau^{k}$ for different $k \in \mathbb{Z}$ are not symplectically isotopic (with compact support), although $\tau^{2}$ is topologically isotopic to the identity.

(4) A Lagrangian embedding of the 3 -sphere into $\mathbb{C P}^{1} \times \mathbb{C}^{2}$ can be defined by

$$
\mathbb{C}^{2} \supset S^{3} \ni\left(z_{1}, z_{2}\right) \longmapsto\left(\left[z_{1}: z_{2}\right],\left(\bar{z}_{1}, \bar{z}_{2}\right)\right) \in \mathbb{C P}^{1} \times \mathbb{C}^{2},
$$

see [2, Example 2.2.8]. Analogous to Example (3), and with $D T^{*} S^{3}$ denoting a small cotangent disc bundle of $S^{3}$ and $B \subset \mathbb{C}^{2}$ a large ball, we can build the symplectic cap

$$
C:=\left(\left(\mathbb{C P}^{1} \times B\right) \backslash D T^{*} S^{3}\right) \cup\left(\left(\mathbb{C P}^{1} \times \mathbb{C}^{2}\right) \backslash\left(\mathbb{C P}^{1} \times B\right)\right) .
$$

Over the first part of $C$ (crossed with $D^{2}$ ) we allow ourselves a generic choice of almost complex structure, on the second part we choose the obvious complex structure, which gives us the holomorphic spheres in the cap.

The intersection argument that we used in the proof of our main theorem in order to show that the spheres in our moduli space are simple no longer applies, since the hypersurface $\{\infty\} \times \mathbb{C}^{2}$ is not contained in $C$. Instead however, we may reason as in Example (2) that a class $H_{2}(\widetilde{W})$ can be regarded as a class in $H_{2}(C)$. From $\tilde{\omega}([F])=\omega_{\mathrm{FS}}([F])=\pi$ (with $F=\mathbb{C P}^{1} \times *$ ), and the observation that $\tilde{\omega}$ takes values in $\pi \mathbb{Z}$ on spherical classes, we conclude that spheres in the class $[F]$ are simple. Also as in Example (2) we see that semi-positivity (which is automatic on $C$ for dimensional reasons) carries over to the relevant 8-dimensional cobordism.

Thus, as in Example (3) we see that the open book with page the cotangent disc bundle of $S^{3}$ and monodromy a $k$-fold Dehn twist $\tau^{k}$ supports a contact structure with a contractible periodic Reeb orbit. The resulting manifolds, analogous to (3), are the Brieskorn manifolds $\Sigma(|k|, 2,2,2,2)$. These have homology $H_{3} \cong \mathbb{Z}_{|k|}$, cf. [18, p. 37]. Hence, in this dimension the Dehn twists $\tau^{k}$ for different $k \in \mathbb{Z}$ are not even topologically isotopic.

Acknowledgements. We are grateful to Sam Lisi for questions and suggestions that prompted the present paper. We thank Emmanuel Giroux for indicating to us how to prove Proposition 2, and Fan Ding for help with a technical point in that 
proof. We thank Janko Latschev for useful discussions. Some parts of this research were done during the workshop 'From Conservative Dynamics to Symplectic and Contact Topology' at the Lorentz Center, Universiteit Leiden, in August 2012. We thank the Lorentz Center and its efficient staff for providing an excellent research environment.

\section{REFERENCES}

[1] C. Abbas, K. Cieliebak and H. Hofer, The Weinstein conjecture for planar contact structures in dimension three, Comment. Math. Helv. 80 (2005), 771-793.

[2] M. Audin, F. Lalonde And L. Polterovich, Symplectic rigidity: Lagrangian submanifolds, Holomorphic Curves in Symplectic Geometry, Progr. Math. 117 (Birkhäuser, Basel, 1994), 271-321.

[3] F. Bourgeois, Ya. Eliashberg, H. Hofer, K. Wysocki and E. Zehnder, Compactness results in symplectic field theory, Geom. Topol. 7 (2003), 799-888.

[4] K. CieliebaK, Subcritical Stein manifolds are split, preprint (2002), arXiv: math/0204351

[5] K. Cieliebak and Ya. Eliashberg, From Stein to Weinstein and Back: Symplectic Geometry of Affine Complex Manifolds, Amer. Math. Soc. Colloq. Publ. 59 (American Mathematical Society, Providence, RI, 2012).

[6] K. Cieliebak and K. Mohnke, Compactness for punctured holomorphic curves, J. Symplectic Geom. 3 (2005), 589-654.

[7] K. Cieliebak and K. Mohnke, Symplectic hypersurfaces and transversality in GromovWitten theory, J. Symplectic Geom. 5 (2007), 281-356.

[8] Ya. Eliashberg, A few remarks about symplectic filling, Geom. Topol. 8 (2004), 277-293.

[9] H. Geiges, An Introduction to Contact Topology, Cambridge Stud. Adv. Math. 109 (Cambridge University Press, Cambridge, 2008).

[10] H. Geiges and K. Zehmisch, How to recognise a 4-ball when you see one, preprint (2011), arXiv: 1104.1543

[11] H. Geiges And K. Zehmisch, Symplectic cobordisms and the Weinstein conjecture, Math. Proc. Cambridge Philos. Soc. 153 (2012), 261-279.

[12] E. Giroux, Convexité en topologie de contact, Comment. Math. Helv. 66 (1991), 637-677.

[13] E. Giroux, Géométrie de contact: de la dimension trois vers les dimensions supérieures, Proceedings of the International Congress of Mathematicians, Vol. II (Higher Education Press, Beijing, 2002), 405-414.

[14] F. Hirzebruch and K. H. Mayer, O(n)-Mannigfaltigkeiten, exotische Sphären und Singularitäten, Lecture Notes in Math. 57 (Springer, Berlin, 1968).

[15] H. Hofer, Pseudoholomorphic curves in symplectizations with applications to the Weinstein conjecture in dimension three, Invent. Math. 114 (1993), 515-563.

[16] M. Hutchings, Taubes's proof of the Weinstein conjecture in dimension three, Bull. Amer. Math. Soc. (N.S.) 47 (2010), 73-125.

[17] M. KluKas, Open books and exact symplectic cobordisms, preprint (2012), arXiv: 1207.5647.

[18] O. van Koert, Open books for contact 5-manifolds and applications of contact homology, Inaugural-Dissertation, Universität zu Köln (2005).

[19] O. van Koert, Contact homology of Brieskorn manifolds, Forum Math. 20 (2008), 317-339.

[20] O. van Koert and K. NiederkrüGer, Open book decompositions for contact structures on Brieskorn manifolds, Proc. Amer. Math. Soc. 133 (2005), 3679-3686.

[21] E. Lerman, Symplectic cuts, Math. Res. Lett. 2 (1995), 247-258.

[22] P. Lisca and G. Matić, Tight contact structures and Seiberg-Witten invariants, Invent. Math. 129 (1997), 509-525.

[23] D. McDuff, Symplectic manifolds with contact type boundaries, Invent. Math. 103 (1991), 651-671.

[24] F. Pasquotto, A brief history of the Weinstein conjecture, Jahresber. Deutsch. Math.Verein., to appear.

[25] P. Seidel, Lectures on four-dimensional Dehn twists, Symplectic 4-Manifolds and Algebraic Surfaces, Lecture Notes in Math. 1938 (Springer, Berlin, 2008), 231-267. 
[26] C. H. Taubes, The Seiberg-Witten equations and the Weinstein conjecture, Geom. Topol. 11 (2007), 2117-2202

[27] A. Weinstein, Symplectic manifolds and their Lagrangian submanifolds, Adv. Math. 6 (1971), 329-346.

[28] A. Weinstein, On the hypotheses of Rabinowitz' periodic orbit theorems, J. Differential Equations 33 (1979), 336-352.

Mathematisches Institut, Universität zu Köln, Weyertal 86-90, 50931 Köln, GerMANY

E-mail address: mdoerner@math.uni-koeln.de

E-mail address: geiges@math.uni-koeln.de

E-mail address: kai.zehmisch@math.uni-koeln.de 